\title{
Abrupt transitions from reinfections in social contagions
}

\author{
Jesús Gómez-Gardeñes, ${ }^{1,2}$ Alessandro S. de Barros, ${ }^{3}$ Suani T.R. Pinho, ${ }^{4}$ and Roberto F.S. Andrade ${ }^{4}$ \\ ${ }^{l}$ Department of Condensed Matter Physics, University of Zaragoza, E-50009 Zaragoza, Spain \\ ${ }^{2}$ Institute for Biocomputation and Physics of Complex Systems (BIFI), University of Zaragoza, E-50018 Zaragoza, Spain \\ ${ }^{3}$ Departamento de Física, Instituto Federal da Bahia, Salvador (Bahia), Brazil \\ ${ }^{4}$ Instituto de Fisica, Universidade Federal da Bahia, Salvador (Bahia), Brazil
}

(Dated: December 10, 2014)

\begin{abstract}
The study of social contagion processes is of utmost importance for understanding the emergence of collective social states. Here we introduce reinfections in the Susceptible-Infected-Recovered model so to incorporate the possibility that an individual that cease its activity (recovered) can resume it due to secondary infections from their active (infected) peers. We show that, when primary infection is less frequent than secondary ones, a typical situation in many social contagion processes, the epidemic transition turns from second to first order. As a consequence, macroscopic collective states can be triggered from the inactive (healthy) regime by a small increment of the primary contagion rate.
\end{abstract}

PACS numbers: 89.65.-s, 89.75.Fb, 89.75.Hc

Contagion processes are at the core of the emergence of many collective social phenomena including the spread of information and gossips, the adoption of beliefs and behaviors, or the massive use of products and innovations [1-3]. The study of such phenomena usually relies in epidemic models $[4,5]$, thus assumming that ideas, products or beliefs spread in a viral way through pairwise contacts between individuals [6-8]. The recent advent of online social networks has amplified the number and coverage of such pairwise contacts, thus reshaping the social contagion patterns [9-12]. As a product, the unfolding of collective actions and protests, such as the Indignados [13] and Occupy Wall Street [14] movements, has turned into global and almost explosive phenomena.

The massive use of these new communication means has challenged the mathematical frameworks aimed at describing contagion processes. In this way, the usual compartmental epidemic models, such as the Susceptible-InfectedSusceptible (SIS) and the Susceptible-Infected-Recovered (SIR) [15], have been recently reformulated to incorporate the dynamical and structural ingredients of the interaction networks observed in real social systems [16-24].

The SIR and SIS models have been intensively studied within the context of non-equilibrium phase transitions and critical phenomena [26-28] as two cornerstones of epidemic modeling [25]. In addition, these are also considered as natural frameworks for the study of social contagion [29-31]. The SIR model assumes that when individuals recover after being infected they cannot catch the disease again. In a social context, this implies that when individuals cease their activity (usage of some product, or spreading any idea or innovation), they are not allowed to resume it. This is not realistic in many real social scenarios in which, for instance, being active means using a product or spreading an idea. In such cases, the SIS model is a more suitable framework as it gets rid off this constraint by considering multiple infections, so that individuals can alternate the active and inactive states.

An important issue that is neglected under the SIS framework is the difference between the primary infection and sec- ondary ones. For instance, think of the dissemination of a new online social network that we start using following the recommendation of a peer. This primary contagion requires us to make an effort due to, for instance, the unavoidable installation and training processes. However, if this contagion is a reinfection, i.e., we resume using the platform after being inactive, the effort invested will be much smaller.

In this Letter we explore the impact that reinfections have on the onset of collective (epidemic) states. To this aim, we transform the SIR model into a SIRI one [32-35] by incorporating secondary infections. As in the SIR case, in the SIRI model individuals can be in three states or compartments: $S$ healthy (ignorant), $I$ infected (active/spreader) and $R$ recovered (inactive). The elementary transitions between these compartments are defined as: (i) $S+I \rightarrow 2 I$ with rate $\lambda$, (ii) $I \rightarrow R$ with rate $\mu$ and (iii) $R+I \rightarrow 2 I$ with rate $\lambda^{\prime}$. Thus, at variance with the SIR model, here recovered (inactive) individuals can become again infected (spreaders/users). However, in general, the reinfection rate is not equal, $\lambda \neq \lambda^{\prime}$, to that corresponding to the primary contagion.

A clear evidence of the strong influence that reinfections have on the epidemic diagram is reported in Fig. 1. There we show the fraction of recovered individuals $r$ as a function of the contagion rate $\lambda$ for $\lambda^{\prime}=0$ and $\lambda^{\prime}=0.15$. The network used is an Erdös-Renyi graph of $N=5 \cdot 10^{3}$ nodes and average degree $\langle k\rangle=6$. For each value of $\lambda$ we have run $10^{2}$ Montecarlo (MC) simulations of the SIRI model as follows. We start by randomly choosing a small fraction, 0.05 , of individuals and set them as infected while the rest of the population is set as healthy. Then we iterate the SIRI dynamics as introduced above for a number of MC steps, until the convergence to a stationary state is reached. In this way each colored point in Fig. 1 represents the final value of $r$ in the steady state of a single realization of the SIRI dynamics.

The case $\lambda^{\prime}=0$ in Fig. 1 corresponds to the usual SIR model (no reinfections are allowed) and thus the curve $r(\lambda)$ shows that the steady value for the fraction of recovered individuals $r$ grows smoothly with $\lambda$ after some critical value $\lambda_{c}$. 


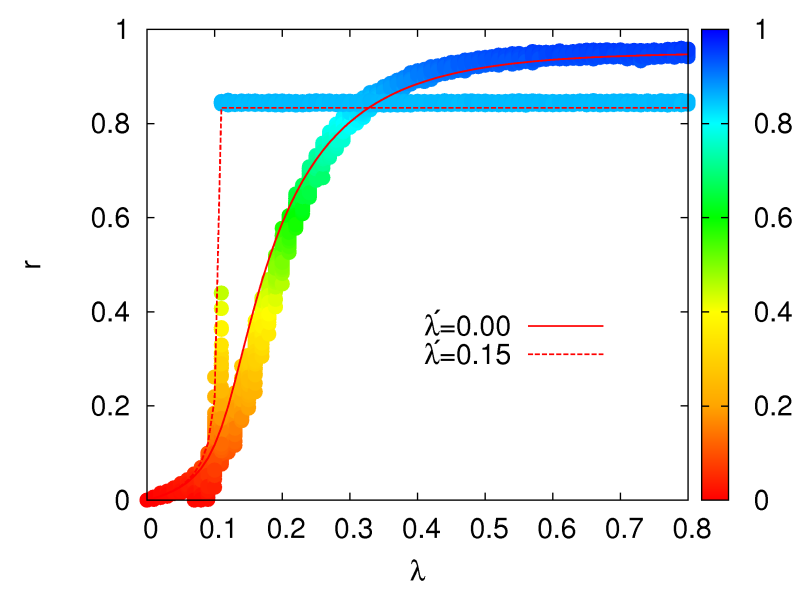

FIG. 1: (color online) Fraction of recovered individuals as a function of $\lambda$. We show two diagrams of the SIRI one (solid curve) corresponding to $\lambda^{\prime}=0$ (SIR model) and another one (dashed curve) where he reinfection rate is set to $\lambda^{\prime}=0.15$. The recovery rate is $\mu=0.8$. The color code indicates (as shown in the color bar) the initial fraction of recovered individuals. The network used is an ER graph with $N=5000$ nodes and average degree $\langle k\rangle=6$.

As usual in the SIR dynamics, for $\lambda^{\prime}=0$ no infected individuals remain in the stationary state so that individuals are either in states $S$ or $R$. In sharp contrast, when reinfections are allowed, $\lambda^{\prime}=0.15$, the transition turns abrupt so that, close to the epidemic onset, a small increment in the contagion rate $\lambda$ triggers a sudden raise in the fraction of recovered individuals. Thus, surprisingly, the introduction of reinfections in the SIR model affects dramatically the nature of its epidemic transition, that changes from second to first order.

The results shown in Fig. 1 provide a flavor of what one gets when MC simulations are carried out for a range of the contagion parameters $\lambda$ and $\lambda^{\prime}$. In Fig. 2 we show the diagrams (a) $r\left(\lambda, \lambda^{\prime}\right)$ and (b) $i\left(\lambda, \lambda^{\prime}\right)$ for the same graph used in Fig. 1. As in Fig. 1, we have plotted all the results obtained in each single realization of the MC dynamics $\left[10^{2}\right.$ realization per each $\left(\lambda, \lambda^{\prime}\right)$ value explored]. From panel (a) we clearly observe the impact of $\lambda^{\prime}$ on the behavior of $r(\lambda)$. Namely, for small values of the reinfection rate $\lambda^{\prime}$ the behavior of $r(\lambda)$ is qualitatively similar to that of the SIR model, i.e., it displays a smooth transition from the healthy (inactive) phase to the epidemic one. The only effect of $\lambda^{\prime}$ in this region is that, for $\lambda>\lambda_{c}$, the fraction of recovered individuals slightly increases with $\lambda^{\prime}$. In this region, the fraction of infected individuals is zero, $i=0$, as in the SIR model (see panel (b)).

When $\lambda^{\prime}$ increases beyond some value $\lambda_{c}^{\prime}$ the picture changes dramatically and the abrupt transition in $r(\lambda)$ shows up. The critical point $\lambda_{c}$ remains almost equal to that of the regime corresponding to small $\lambda^{\prime}$. However, at $\lambda_{c}$ the abrupt transition drives the system from the healthy to another regime, where a macroscopic part of the population was affected by the disease. At variance with the region corresponding to small reinfection rates, here the fraction of recovered individuals at $\lambda>\lambda_{c}$ is completely independent of the value $\lambda$, and thus remain constant for $\lambda>\lambda_{c}$ (as it was shown in Fig. 1 for $\lambda^{\prime}=0.15$ ). In addition, panel (b) shows another important novelty in relation to the SIR model, since infected and recovered individuals coexist in the steady state when $\lambda>\lambda_{c}$. Moreover, the curves $i(\lambda)$ reveal that the phase transitions at $\lambda_{c}$ for the fraction of infected individuals are also abrupt.

To gain a deeper insight about the origin of the abrupt transition observed via MC simulations, we considered the Markovian evolution equations of the SIRI model on a complex network. This approach, originally introduced for the analysis of the epidemic SIS model [36-38], deals with the the probabilities that each individual $j$ is infected or recovered at time $t: \mathbf{p}(t)=\left\{p_{j}(t)\right\}$ and $\mathbf{r}(t)=\left\{r_{j}(t)\right\}(j=1, \ldots, N)$ respectively. Given the contagion, $\lambda$ and $\lambda^{\prime}$, and recovery, $\mu$, rates, we can write the time-discrete evolution equations for the former two sets of probabilities as:

$$
\begin{aligned}
p_{j}(t+1)= & p_{j}(t)(1-\mu)+r_{j}(t) q_{j}^{R I}(t) \\
& +\left[1-p_{j}(t)-r_{j}(t)\right] q_{j}^{S I}(t), \\
r_{j}(t+1)= & p_{j}(t) \mu+r_{j}(t)\left[1-q_{j}^{R I}(t)\right] .
\end{aligned}
$$

Here $q_{j}^{S I}(t)$ and $q_{j}^{R I}(t)$ are the probabilities that agent $j$ becomes infected at time $t$, provided it is susceptible or recovered respectively. The expressions for these probabilities are:

$$
\begin{gathered}
q_{j}^{S I}(t)=1-\prod_{l=1}^{N}\left(1-\lambda A_{j l} p_{l}(t)\right), \\
q_{j}^{R I}(t)=1-\prod_{l=1}^{N}\left(1-\lambda^{\prime} A_{j l} p_{l}(t)\right) .
\end{gathered}
$$

where $\mathbf{A}=\left\{A_{j l}\right\}$ is the $N \times N$ adjacency matrix of the underlying graph, defined as $A_{j l}=1$ if agents $j$ and $l$ are connected and $A_{j l}=0$ otherwise.

Of particular importance are the solutions of Eqs. (1) and (2) corresponding to stationary distributions for the probabilities of being infected and recovered, $\left\{p_{j}^{*}\right\}$ and $\left\{r_{j}^{*}\right\}$. These solutions can be obtained by iterating Eqs. (1) and (2) from the set of initial conditions, $\mathbf{p}(0)$ and $\mathbf{r}(0)$, until the probabilities converge to their stationary value, $\mathbf{p}^{\star}$ and $\mathbf{r}^{\star}$. To compare with MC simulations we compute the fraction of infected (recovered) individuals as: $i=\sum_{j=1}^{N} p_{j}^{\star} / N\left(r=\sum_{j=1}^{N} r_{j}^{\star} / N\right)$. The great accuracy of the solutions of the Markovian evolution equations is shown (solid and dashed curves) in Fig. 1. Remarkably, the solutions of the Markovian equations fully agree with those obtained via MC simulations and, importantly, they reproduce the abrupt transition when reinfections $\left(\lambda^{\prime}=0.15\right)$ are at work.

We now analyze the fixed points of the Markovian Eqs. (1) and (2) to understand the roots of the different behaviors of $r(\lambda)$ and $i(\lambda)$ shown in Figs. 1 and 2. By imposing the stationary contiditon in Eqs. (1) and (2), $p_{j}(t+1)=p_{j}(t)=p_{j}^{*}$ and $r_{j}(t+1)=r_{j}(t)=r_{j}^{*}$, we obtain:

$$
\begin{aligned}
& p_{j}^{*}=p_{j}^{*}(1-\mu)+\left(1-p_{j}^{*}-r_{j}^{*}\right) q_{j}^{S I^{*}}+r_{j}^{*} q_{j}^{R I^{*}}, \\
& r_{j}^{*}=p_{j}^{*} \mu+r_{j}^{*}\left(1-q_{j}^{R I^{*}}\right) .
\end{aligned}
$$



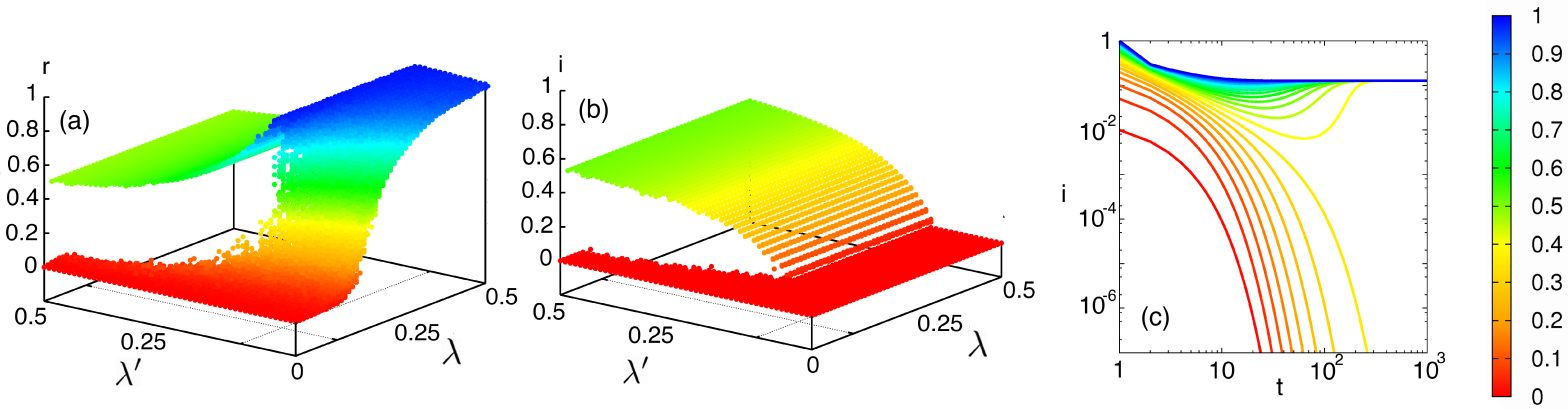

FIG. 2: (color online) Fraction of (a) recovered, $r$, and (b) infected, $i$, individuals as a function of $\lambda$ and $\lambda^{\prime}$. As observed the transitions $r(\lambda)$ and $i(\lambda)$ are discontinuous when $\lambda^{\prime}$ is large enough. Note that the plots show $10^{2}$ points for each value $\left(\lambda, \lambda^{\prime}\right)$. Each point represents the average steady value of $r$ and $i$ in each of the $10^{2}$ MC realizations of the SIRI dynamics. Panel (c) shows the evolution of the fraction of infected individuals, $i$, as obtained from Eqs. (1) and (2). The values of the infection, reinfection and recovery rates are fixed to $\lambda=0.08<\lambda_{c}$, $\lambda^{\prime}=0.15>\lambda_{c}\left(\lambda_{c}=0.118\right)$ and $\mu=0.8$ respectively. The color of each point in (a) and (b) and each curve in (c) indicates, respectively, the value of $r, i$ and the initial fraction of infected individuals as indicated in the color bar (right). The network is the same as in Fig. 1.

By adding these equations we derive the following necessary condition for the existence of such a stationary solution:

$$
\left(1-p_{j}^{*}-r_{j}^{*}\right) q_{j}^{S I^{*}}=0,
$$

which is satisfied when either $r_{j}^{*}+p_{j}^{*}=1$ or $q_{j}^{S I^{*}}=0$ holds. Note that the second condition is equivalent (for $\lambda>0$ ) to $p_{j}=0$ and thus it applies for the SIR-like regime (small values of $\lambda^{\prime}$ ) whereas the first condition appears to hold (as observed from Fig. 2) in the large $\lambda^{\prime}$ region, i.e., where only infected and recovered individuals coexist. Thus, there is no stationary solution containing a non-vanishing fraction of the three compartments.

In the large $\lambda^{\prime}$ region we can evaluate the stationary probabilities $p_{j}^{*}$ (and $r_{j}^{*}$ ) by substituting $r_{j}^{*}=1-p_{j}^{*}$ in Eq. (6):

$$
\mu p_{j}^{*}=\left(1-p_{j}^{*}\right) q_{j}^{R I^{*}} .
$$

The above equation is equivalent to that of the stationary probabilities for the infected individuals in the SIS model, when replacing the compartment $S$ by $R$. Thus we term as RIR the large $\lambda^{\prime}$ regime. Note also that the value of $p_{j}^{*}$ in the RIR regime is independent of $\lambda$ since the expression of $q_{j}^{R I}$, Eq. (4), only depends on $\lambda^{\prime}$. This is again in agreement with the MC results shown in Fig. 2 for the RIR regime where the stationary values $i$ and $r$ only depend on $\lambda^{\prime}$.

Now we analyze the domain of the RIR regime. Considering that $\lambda^{\prime}$ enhances the passage from $R$ to $I$, the values of the probabilities $p_{j}^{*}$ are expected to increase with $\lambda^{\prime}$. Thus, we now check what is the minimum value of $\lambda^{\prime}$ so to have an infinitesimally small fraction of infected individuals (coexisting with a macroscopic fraction of recovered ones), i.e., the threshold $\lambda_{c}^{\prime}$ for the RIR regime. By considering $p_{j}^{*}=\epsilon_{j}$ we get $q_{j}^{R I^{*}} \simeq \lambda^{\prime} \sum_{l=1}^{N} A_{j l} \epsilon_{l}$ and thus neglecting terms of second order in $\epsilon_{j}$ in Eq. (8) we obtain:

$$
\frac{\mu}{\lambda^{\prime}} \vec{\epsilon}=\mathbf{A} \vec{\epsilon} .
$$

This yields that the threshold for the existence of the RIR regime $\left(r_{j}^{*}+p_{j}^{*}=1\right)$ is equal to:

$$
\lambda_{c}^{\prime}=\frac{\mu}{\max \{\Lambda(\mathbf{A})\}},
$$

where $\max \{\Lambda(\mathbf{A})\}$ is the maximum eigenvalue of the adjacency matrix A. Above this value of $\lambda^{\prime}$ the solution $p_{j}^{*}+r_{j}^{*}=$ 1 exists. This threshold is again in agreement with the numerical results shown in Fig. 2 as noted by the (dashed) line pointing out the value $\lambda_{c}^{\prime}=0.118$ obtained for the network used in the MC simulations.

Interestingly, the independence of Eq. (10) with respect to $\lambda$ points out that the whole region $\lambda^{\prime}>\lambda_{c}^{\prime}$ should display RIR steady states, $r_{j}+p_{j}=1$. On the contrary, as noted above, in Fig. 2 there is a region of small values of $\lambda\left(\lambda<\lambda_{c}\right)$ in which the fraction of infected individuals is zero and that of recovered ones is infinitesimally small. This suggest that, in this region, bistability between the Healthy and the RIR phases takes place. However, due to the small fraction of initially infected agents used in the MC simulations in Figs. 2.a and 2.b the system is driven directly towards the healthy regime. To illustrate the bistability we show in Fig. 2.c the time evolution $i(t)$ for different initial conditions and with contagion and reinfection rates lying in the bistable phase $(\lambda=0.08$ and $\left.\lambda^{\prime}=0.15\right)$. In this case it becomes clear that an initial condition containing more than $40 \%$ of infected individuals will end up in the RIR regime, otherwise the dynamics evolves towards the Healthy phase.

Finally, for $\lambda^{\prime}<\lambda_{c}^{\prime}$ the RIR regime is no longer valid and the steady condition that applies to satisfy Eq. (7) is $p_{j}=0$. This condition is compatible with the usual steady states of the SIR in its two possible phases: (i) Healthy phase, $r_{j}^{*}=r_{j}(0)$ for $\lambda<\lambda_{c}$, and (ii) Epidemic phase $r_{j}^{*}>r_{j}(0)$. The critical value $\lambda_{c}$ can be evaluated by imposing $p_{j}(1)=p_{j}(0)=\epsilon_{j} \ll$ 1 in Eq. (1). The analysis was done in [39] yielding a critical value $\lambda_{c}$ equal to that of $\lambda_{c}^{\prime}$, Eq. (10). Thus for $\lambda^{\prime}<\lambda_{c}^{\prime}$ and $\lambda<\lambda_{c}^{\prime}\left(\lambda>\lambda_{c}^{\prime}\right)$ the system lies in the Healthy (Epidemic) phase. 


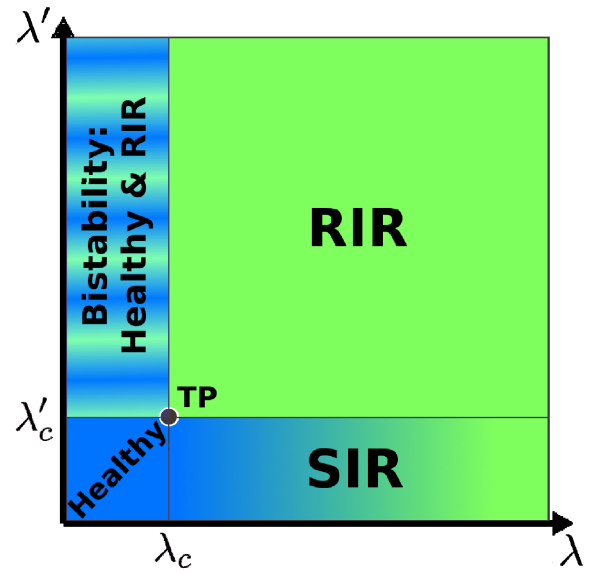

FIG. 3: (color online) Schematic representation of the phase diagram of the SIRI model. Note the existence of a Triple point (TP) separating the Healthy, SIR and RIR phases.

Summarizing, from the theoretical side, we have shown that the addition of reinfections to the SIR model (SIRI model) changes dramatically its critical properties in a way that an abrupt phase transition from the healthy phase to the epidemic one shows up. In Fig. 3 we show a schematic picture of the phase diagram of the SIRI model. For low values of the reinfection probability $\lambda^{\prime}$ we find the two typical phases of the usual SIR model: Healthy phase for $\lambda<\lambda_{c}$ and the Epidemic phase for $\lambda>\lambda_{c}$ in which the number of recovered individuals increases smoothly with $\lambda$ whereas the number of infected individuals vanishes. On the contrary, for $\lambda^{\prime}>\lambda_{c}^{\prime}$ the situation is totally different. For $\lambda>\lambda_{c}$ susceptible individuals dissapear from the population (the RIR regime) as a consequence of the non-zero steady number of infected individuals due to the reinfections of recovered individuals. On the other hand, if the the value of $\lambda$ is small enough the RIR regime cannot be activated unless the initial fraction of infected individuals is large enough. Thus, in this region there is a coexistence (bistability) of the Healthy and RIR phases.

The most striking result is that for $\lambda^{\prime}>\lambda_{c}^{\prime}$ the transition between the Healthy phase and the RIR regime is abrupt. In this way, a small change in the contagion probability $\lambda$ drives the system from a null invasion of the disease to a macroscopic one, being the corresponding transitions for both the fraction of infected and recovered individuals of first order. Interestingly, as shown in Fig. 3, the phase diagram includes a Triple Point (TP) $\left(\lambda_{c}, \lambda_{c}^{\prime}\right)$ in which the three possible phases (Healthy, Epidemic SIR and Epidemic RIR) coexist [40]. The fact that $\lambda_{c}=\lambda_{c}^{\prime}$ indicates that, in the case that primary and secondary infections are equally probable, $\lambda=\lambda^{\prime}$, the epidemic onset lies just at the TP. In this particular case the onset is abrupt for $r(\lambda)$ and smooth for $i(\lambda)$ so that the system displays a kind of mixed-order phase transition as a consequence of being placed just on the TP in which two kinds of phase transitions coincide. This result is presented in the Supplemental [41] accompanying this letter.
On the practical side, our results have important consequences for the study of real spreading scenarios in which reinfections play a key role in the long term dynamics. As noted in the introductory part, the most paradigmatic scenario where reinfections are more prone than primary ones is that of social contagion of products, innovations, beliefs or ideas, for which primary adoptions by ignorants are less probable than secondary ones. In the language of the epidemic model this feature implies that $\lambda^{\prime}>\lambda$, thus making the transition towards collective contagion abrupt so that a successful dissemination of an ida or product becomes viral without almost any early warning.

JGG is supported by the Spanish MINECO through the Ramón y Cajal program and Projects FIS2011-25167 and FIS2012-38266-C02-01, the Comunidad de Aragón (FENOL group) and the Brazilian CNPq through the grant PVE of the Ciencias Sem Fronteiras program. ASB, STRP, RFSA are partially supported by FAPESB (contract number PNX 0006/2009). ASB and STRP are also supported by CNPQ (INCT-CITECS); and RFSA, by CNPQ (INCT-SC).

[1] D. Centola, Science 329, 1194 (2010).

[2] N. A. Christakis, and J. H. Fowler, New England J. Med. 357, 370 (2007).

[3] T. W. Valente, Soc. Networks 18, 69 (1996).

[4] W. Goffman, and V. A. Newill, Nature 204, 225 (1964).

[5] D. J. Daley, and D. G. Kendall, Nature, 204, 1118 (1964).

[6] E.M. Rogers, Diffusion of Innovations, (Simon and Schuster, New York, 2010).

[7] J. Berger, and K.L. Milkman, Journal of Marketing Research 49, 192 (2012).

[8] J. Berger, Contagious: why things catch on (Simon \& Schuster, 2014).

[9] M.R. Subramani, and B. Rajagopalan, Communications of the ACM, 46, 307 (2003).

[10] J. P. Onnela, Proc. Natl. Acad. Sci. USA 104, 7332 (2007).

[11] J. Yang, and J. Leskovec, 2010 IEEE International Conference on Data Mining, 599 (2010).

[12] G. Miritello, R. Lara, M. Cebrian, and E. Moro, Sci. Rep. 3, 1950 (2013).

[13] J. Borge-Holthoefer et al., PLoS One 6, e23883 (2011).

[14] M.D. Conover, E. Ferrara, F. Menczer, and A. Flammini, PLoS One 8, e64679 (2013).

[15] J. D. Murray, Mathematical Biology,Springer-Verlag, Germany, Berlin (2002).

[16] R. Pastor-Satorras, and A, Vespignani, Phys. Rev. Lett. 86, 3200 (2001).

[17] M. Kuperman and G. Abramson, Phys. Rev. Lett. 86, 2909 (2001).

[18] M.J. Keeling and K.T.D. Eames, J. Roy. Soc. Interface 2, 295 (2005).

[19] T. Gross, C.J.D. D’Lima, B. Blasius, Phys. Rev. Lett. 96, 208701 (2006).

[20] V. Colizza, and A. Vespignani, Phys. Rev. Lett. 99, 148701 (2007).

[21] A.V. Goltsev, S.N. Dorogovtsev, J.G. Oliveira, J.F.F. Mendes, Phys. Rev. Lett. 109, 128702 (2012).

[22] A. Vespignani, Nature Phys. 8, 32 (2012). 
[23] C. Granell, S. Gómez, and A. Arenas, Phys. Rev. Lett. 111, 128701 (2014).

[24] S.Y. Liu, N. Perra, M. Karsai, and A. Vespignani, Phys. Rev. Lett. 112, 118702 (2014).

[25] M.J. Keeling and P. Rohani, Modeling Infectious Diseases in Human and Animals (Princeton University Press, Princeton, 2008).

[26] J. Marro and R. Dickman, Nonequilibrium Phase Transitions in Lattice Models, (Cambridge University Press, Cambridge, 1990).

[27] P. Grassberger, Math. Biosc. 63, 157 (1983).

[28] T. Tomé, and R.M. Ziff, Phys. Rev. E 82, 051921 (2010).

[29] C. Castellano, S. Fortunato, V. Loreto, Rev. Mod. Phys. 81, 591 (2009).

[30] M. Draief and L. Massoulié, Epidemics and Rumours in Complex Networks, London Mathematical Society Lecture Notes Series 369 (2010).

[31] A.L. Hill, D.G. Rand, M.A. Nowak, N.A. Christakis, PLoS Comput Biol 6, e1000968 (2010).

[32] D. Tudor, SIAM Rev. 32, 136 (1990).

[33] H.N. Moreira, and Y. Wang, SIAM Rev. 39, 496 (1997).
[34] P. Rodrigues, M.G.M. Gomes, A. Margheri, C. Rebelo. J. Theor. Biol. 259, 280 (2009).

[35] A.S. de Barros, Stochastic Dynamics for Modelling Reinfection Effect on Directed Transmittes disease, (Universidade Federal da Bahia, Salvador, Brazil, 2013) (in portuguese).

[36] S. Gómez, A. Arenas, J. Borge-Holthoefer, S. Meloni and Y. Moreno, EPL 89, 38009 (2010).

[37] B. Guerra and J. Gómez-Gardeñes, Phys. Rev. E 82, 035101(R) (2010).

[38] S. Gómez, J. Gómez-Gardeñes, Y. Moreno, and A. Arenas, Phys. Rev. E 84, 036105 (2011).

[39] M. Youssef, and C. Scoglio, J. Theor. Biol. 283, 136 (2011).

[40] Note that the TP is associated to non-equilibrium phase transitions, at variance with the usual TP found in equilibrium thermodynamics.

[41] See Supplemental Material at http://link.aps.org/supplemental/xxx for the curves $r(\lambda)$ and $i(\lambda)$ obtained through $\mathrm{MC}$ simulations and by solving the Markovian evolution. 